\title{
OA-006 COMPARATIVE EVALUATION OF GENOTYPE MTBDRPLUS VERSION 2 AND GENE XPERT MTB/RIF ASSAYS TO DETECT MYCOBACTERIUM TUBERCULOSIS AND RESISTANCE GENE PATTERNS IN GABON
}

\author{
Abraham Alabi, ${ }^{1}$ Francis Foguim, ${ }^{1}$ Jeyaraj Sankarganesh, ${ }^{2}$ Ellen Bruske, ${ }^{2}$ \\ Arnault Mfoumbi, ${ }^{1}$ Chester Mevyann, ${ }^{1}$ Ayola Adegnika, ${ }^{1}$ Bertrand Lell, ${ }^{1}$ \\ Katharina Kranzer, ${ }^{3}$ Peter Kremsner, ${ }^{2}$ Martin Grobusch ${ }^{4}$. ${ }^{1}$ CERMEL, Gabon; ${ }^{2}$ ITM \\ Tübingen, Germany, Germany; ${ }^{3}$ NRC for Mycobacteria, Germany; ${ }^{4} A M C$, The \\ Netherlands
}

\subsection{6/bmjgh-2016-000260.15}

Background Tuberculosis (TB) remains a major cause of morbidity and mortality in Africa. A major challenge of TB diagnosis is slow growth of its causative agent, Mycobacterium tuberculosis complex (MTBC). WHO has endorsed the application of molecular methods to rapidly diagnose TB and detect drug resistance mutations in MDR-TB. We hereby evaluate the efficacy of the GenXpert MTB/RIF and the GenoType MTBDRplus, using culture as gold standard.

Methods We applied the GeneXpert MTB/RIF (Cepheid) and the Genotype MTBDRplus (Hain Life Sciences, Germany) to compare between molecular and standard traditional methods (smear microscopy and culture). In total, 246 consecutive sputa samples from suspected TB cases (individuals) in Lambarene and surrounding villages were analysed. The molecular methods confirm MTBC and detect resistant mutations in the rpoB, katG and inh genes corresponding to rifampicin (RIF) and isoniazid (INH), respectively.

Results Of the 193 samples available for analysis, 51 were positive and 142 were negative by culture. The overall sensitivity of GeneXpert compared to culture was $86.3 \%$ and the specificity was 93.7\%. The sensitivity and specificity of Genotype MTBDRplus compared to culture were $82.5 \%$ and $95.8 \%$. Rifampicin-resistant strains determined by standard drug susceptibility testing (DST) were 100\% identified by GeneXpert and $83.3 \%$ by Genotype MTBDRplus. All the rifampicin-resistant strains were also exhibiting the high level resistance against highlevel isoniazid corresponding to katG genes. Genotype MTBDRplus identified two isolates carrying only mutations to low-level isoniazid resistance.

Conclusions This comparative study has established a strong correlation between the GeneXpert and the MTBDRplus assays for the rapid diagnosis of multi-drug resistant TB (MDR-TB); as well as with drug susceptibility testing by standard culture method. Our findings further strengthen the WHO recommendation for the universal implementation of molecular tests in order to enhance the rapid diagnosis of TB and early initiation of treatment in confirmed cases. 